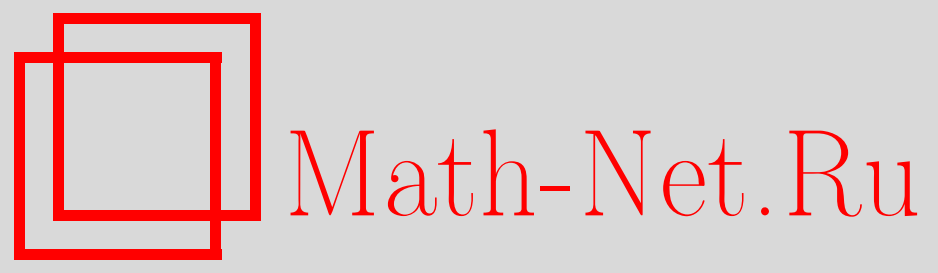

Ю. В. Жуков, О точности нормальной аппроксимации для плотностей сумм независимых одинаково распределенных случайных величин, Теория вероятн. и ее примен., 1999, том 44, выпуск 4, 853-861

DOI: https://doi.org/10.4213/tvp1070

Использование Общероссийского математического портала Math-Net.Ru подразумевает, что вы прочитали и согласны с пользовательским соглашением

http://www.mathnet.ru/rus/agreement

Параметры загрузки:

IP : 52.23 .180 .231

26 апреля 2023 г., 04:06:07

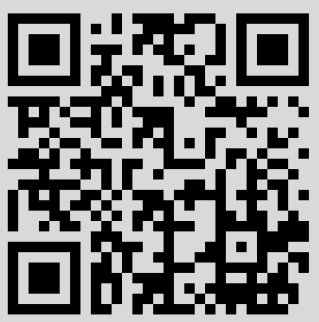




\title{
О ТОЧНОСТИ НОРМАЛЬНОЙ АППРОКСИМАЦИИ ДЛЯ ПЛОТНОСТЕЙ СУММ НЕЗАВИСИМЫХ ОДИНАКОВО РАСПРЕДЕЛЕННЫХ СЛУЧАЙНЫХ ВЕЛИЧИН
}

\begin{abstract}
Уточняется структура неравномерной оценки скорости сходимости в локальной центральной предельной теореме для плотностей сумм независимых одинаково распределенных случайных величин. Абсолютные постоянные указаны в явном виде.
\end{abstract}

Ключевые слова и фразы: локальная предельная теорема, неравномерные оценки, метрика $L_{p}$.

1. Введение. Пусть $X_{1}, X_{2}, \ldots$ - независимые одинаково распределенные случайные величины (с.в.) с $\mathbf{E} X_{1}=0, \mathbf{D} X_{1}=1, \mathbf{E}\left|X_{1}\right|^{3} \equiv \beta^{3}$. Предположим, что с.в. $X_{1}$ имеет плотность $p(x)$. Плотность нормированной суммы $S_{n}=\left(X_{1}+\cdots+X_{n}\right) / \sqrt{n}$ и ее характеристическая функция (х.ф.) будут обозначаться $p_{n}(x)$ и $f_{n}(t)$ соответственно. В данной статье мы приводим неравномерну ю оценку точности приближения плотности нормированной суммы независимых одинаково распределенных случайных величин плотностью $\varphi(x)$ стандартного нормального закона. При этом делаются стандартные предположения о том, что $\beta^{3}<\infty$, а общая плотность $p(x)$ слагаемых ограничена некоторым числом $A$ :

$$
A \equiv \sup _{x} p(x)<\infty \text {. }
$$

Сразу заметим, что при приведенных выше условиях на моменты с.в. $X_{1}$ число $A$ не может быть сколь угодно близким к нулю. Более того, используя результаты работы Ю. В. Прохорова [4], можно показать, что $12 A^{2}>1$. Обозначим $\Delta(x)=\left|p_{n}(x)-\varphi(x)\right|$.

Данная работа отнюдь не первая в этом направлении. Еще в середине 60-х годов C.X. Сираждиновым и его учениками наряду с равномерной оценкой для $\Delta(x)$ была получена неравномерная оценка вида

$$
\Delta(x) \leqslant C \frac{\beta^{15} \max \left(1, A^{7}\right)}{\sqrt{n}\left(1+|x|^{3}\right)},
$$

где $C>0$ - абсолютная постоянная. Эта оценка также приведена в широко известной книге В.В. Петрова [3]. Однако, если по числу слагаемых $n$ и аргументу плотности $x$ оценка (1) имеет традиционные для указанных выше моментных предположений порядки убывания, то ее зависимость от других параметров - $\beta^{3}$ и $A-$ далека от оптимальной. Сопоставление оценки (1) с критерием сходимости в локальной центральной предельной теореме, согласно которому для равномерной сходимости $p_{n}(x)$ к $\varphi(x)$ необходимо и достаточно, чтобы существовало некоторое $N$, при котором $p_{N}(x)$ ограничена, наводит на мысль о том, что роль константы $A$ в оценке (1) сильно преувеличена. Действительно, в работе В.Ю. Королева и Ю.В. Жукова [9] удалось получить равномерную оценку

$$
\sup _{x} \Delta(x) \leqslant 0.4013 \frac{\beta^{3}}{\sqrt{n}}+\Delta_{n}^{(1)}(\beta)+\Delta_{n}^{(2)}(A, \beta),
$$

где

$$
\begin{aligned}
& \Delta_{n}^{(1)}(\beta)= 0.7958 \frac{\beta}{n^{1 / 6}} \exp \left\{-\frac{n^{1 / 3}}{3 \beta^{2}}\right\} \\
& \Delta_{n}^{(2)}(A, \beta)=\inf _{0<\gamma<1 \pi^{-3}<\delta<1} \inf \frac{A^{2} \beta^{3}}{(1-\delta)^{3 / 2}}\left[\frac{2.22}{\delta^{1 / 3} \sqrt{1-\gamma}}+\frac{9.43}{\sqrt{n}(1-\delta)^{3 / 2}}\right] \\
& \times\left(1-\frac{(1-\delta)^{3}}{12 \pi^{2} A^{2} \beta^{6}}\right)^{\gamma n}
\end{aligned}
$$

* Московский государственный университет им. М. В. Ломоносова, факультет вычислительной математики и кибернетики, Воробьевы горы, 119899 Москва, Россия. 
а для случая симметричных слагаемых также и оценку величины $x^{2} \Delta(x)$, имеюшую структуру, аналогичную оценке (2). Несложно видеть, что, полагая $\gamma=\frac{1}{2}, \delta=0.05$, для величины $\Delta_{n}^{(2)}(A, \beta)$ можно легко получить оценку

$$
\Delta_{n}^{(2)}(A, \beta) \leqslant 16.99 A^{2} \beta^{3}\left(1-\frac{1}{14 \pi^{2} A^{2} \beta^{6}}\right)^{n / 2} .
$$

Цель данной работы - продолжая исследования, начатые в работе [9], для случая не обязательно симметричных слагаемых получить неравномерну ю оценку для $\Delta(x)$, имеющуго традиционный порядок убывания по $x$, со структурой, сходной с оценкой (2). Тем самым мы довольно существенно уточним оценку (1), показав, что на самом деле те члены мажоранты, которые зависят от $A$, убывают по $n$ по крайней мере экспоненииально быстро. Это хорошо согласуется с упомянутым выше критерием сходимости плотностей нормированных сумм к нормальной плотности. Далее, часть предлагаемой в данной статье мажоранты для величины $|x|^{3} \Delta(x)$, не зависящая от $A$, разбивается на два слагаемых: одно из них имеет вид const $\beta^{3} n^{-1 / 2}$, привычный для оценок скорости сходимости в интегральной центральной предельной теореме, второе же имеет более высокий порядок по $\beta$, но по $n$ убывает существенно быстрее. Мы приводим явные значения абсолютных постоянных, входящих в оценки, указываем асимптотические верхние оценки для констант и уточняем оценки для величины

$$
\left\|p_{n}(x)-\varphi(x)\right\|_{p}=\left(\int_{-\infty}^{\infty}\left|p_{n}(x)-\varphi(x)\right|^{p} d x\right)^{1 / p},
$$

полученные в статье [9]. Последний результат полезен для построения оценок скорости сходимости в центральной предельной теореме в метрике полной вариации.

2. Основные результаты.

Теорема 1. При всех $n \geqslant 8 \beta^{6}$ в сделаяных выше предположениях для любого $x \in \mathbf{R}$ справедливо неравенство

$$
|x|^{3} \Delta(x) \leqslant 30.7536 \frac{\beta^{3}}{\sqrt{n}}+\Delta_{n}^{(5)}(\beta)+\Delta_{n}^{(6)}(A, \beta),
$$

$2 \partial e$

$$
\begin{aligned}
\Delta_{n}^{(5)}(\beta)= & 37.2623 \frac{\beta^{3}}{n^{5 / 6}}+\beta \varphi\left(\sqrt{\frac{2}{3}} \frac{n^{1 / 6}}{\beta}\right) \\
& \times\left[1.7867 n^{4 / 3}+5.4876 n^{1 / 3}+3.9895+1.3679 \beta^{3} n^{-2 / 3}\right], \\
\Delta_{n}^{(6)}(A, \beta)= & \inf _{0<\gamma<1 \pi^{-3}<\delta<1} \inf _{(1-\gamma)(1-\delta)^{6}} \frac{A^{4} \beta^{9}}{\left(1-\gamma 95+\frac{211 A}{(1-\gamma) \delta^{4 / 3}}\right]} \\
& \times\left(1-\frac{(1-\delta)^{3}}{12 \pi^{2} A^{2} \beta^{6}}\right)^{\gamma n} .
\end{aligned}
$$

З а м е ч а н и е 1. Очевидно, что $\Delta_{n}^{(5)}(\beta)=o\left(n^{-1 / 2}\right), \Delta_{n}^{(6)}(A, \beta)=o\left(n^{-\nu}\right)$, каким бы ни было $\nu>0$. Более того, полагая $\gamma=\frac{1}{2}, \delta=0.05$, легко получить оценку

$$
\Delta_{n}^{(6)} \leqslant 2.7208 A^{4} \beta^{9}(795+22910 A)\left(1-\frac{1}{14 \pi^{2} A^{2} \beta^{6}}\right)^{n / 2} .
$$

Наличие в выражении (7) свободных параметров $\gamma$ и $\delta$ позволяет путем чпсленной оптимизации получать конкретные значения мажоранты при данных $A, \beta^{3}$ и $n$. Тем не менее, кратко остановимся на аналитической оценке «оптимальных» значений параметров $\gamma$ и $\delta$. Определяющую роль при изучении порядка величины $\Delta_{n}^{(6)}$ играет параметр $\gamma$. Поэтому, реализуя минимаксный подход, вначале мы будем искать минимум по $\gamma$ супремума по $n$ выражения под знаками инфимумов в формуле (7) при 
фиксированном значении $\delta$. Очевидно, что при $n \geqslant 8 \beta^{6}$

$$
\begin{aligned}
& \frac{A^{4} \beta^{9}}{(1-\gamma)(1-\delta)^{6}}\left[795+\frac{211 A}{(1-\gamma) \delta^{4 / 3}}\right]\left(1-\frac{(1-\delta)^{3}}{12 \pi^{2} A^{2} \beta^{6}}\right)^{\gamma n} \\
& \leqslant \frac{A^{4} \beta^{9}}{(1-\delta)^{6}}\left[795+\frac{211 A}{\delta^{4 / 3}}\right]\left(1-\frac{(1-\delta)^{3}}{12 \pi^{2} A^{2} \beta^{6}}\right)^{8 \beta^{6} \gamma}(1-\gamma)^{-2} .
\end{aligned}
$$

Легко убедиться, что минимум правой части этого неравенства по $\gamma$ достигается при

$$
\gamma=\gamma\left(A, \beta^{3}, \delta\right)=1-\left[4 \beta^{6}\left|\ln \left(1-\frac{(1-\delta)^{3}}{12 \pi^{2} A^{2} \beta^{6}}\right)\right|\right]^{-1} .
$$

Теперь ясно, что $\sup _{\pi^{-3}<\delta<1} \gamma\left(A, \beta^{3}, \delta\right)=\gamma\left(A, \beta^{3}, \pi^{-3}\right)$.

Доказательство теоремы 1 мы предварим одним вспомогательным утверждением.

Лемма 1. Для любого $n \geqslant 8 \beta^{6}$ в интервале $|t| \leqslant n^{1 / 6} / \beta$ верно неравенство

$$
\begin{aligned}
& \left|f_{n}^{\prime \prime \prime}(t)-\left(3 t-t^{3}\right) e^{-t^{2} / 2}\right| \\
& \leqslant \frac{\beta^{3}}{\sqrt{n}}\left[0.36\left(t^{6}+3 t^{4}\right) \exp \left\{-0.3779 t^{2}\right\}+\left(3.429 t^{4}+10.7775 t^{2}+6.5559\right) e^{-t^{2} / 3}\right] \\
& \quad+\frac{\beta^{3}}{n^{5 / 6}}\left(1.7145 t^{4}+15.1566 t^{2}+41.9552\right) e^{-t^{2} / 3} .
\end{aligned}
$$

Д о к а 3 а т е л с с т о. Всюду далее х.ф. с.в. $X_{1}$ будет обозначаться $f(t)$. Так как $\mathbf{E} X_{1}=0, \mathbf{D} X_{1}=1,|t| \leqslant n^{1 / 6} / \beta, \beta \geqslant 1$ и $n \geqslant 8$, то

$$
\left|f\left(\frac{t}{\sqrt{n}}\right)-1\right| \leqslant \frac{t^{2}}{2 n} \leqslant \frac{1}{2 n^{2 / 3}}<0.1251, \quad\left|f\left(\frac{t}{\sqrt{n}}\right)\right|>0.8749 .
$$

Поэтому при указанных $t$ определена функция $h(t)=t^{2} / 2+n \ln f(t / \sqrt{n})$. Используя представление $f^{(n)}(t)=i^{n} \int_{-\infty}^{\infty} e^{i t x} x^{n} p(x) d x$ и хорошо известное неравенство (см., например, $[7$, с. 576$])$, имеем

$$
\begin{aligned}
\left|f^{\prime}(t)-f^{\prime}(0)-t f^{\prime \prime}(0)\right| & \leqslant\left|\int_{-\infty}^{\infty} i x\left(e^{i t x}-1-i t x\right) p(x) d x\right| \\
& \leqslant \int_{-\infty}^{\infty}|x|\left|e^{i t x}-1-i t x\right| p(x) d x \leqslant \int_{-\infty}^{\infty}|x| \frac{|t x|^{2}}{2} p(x) d x \leqslant \beta^{3} \frac{t^{2}}{2} .
\end{aligned}
$$

Таким образом, производную х.ф. $f$ мы можем представить в следующем виде:

$$
f^{\prime}\left(\frac{t}{\sqrt{n}}\right)=-\frac{t}{\sqrt{n}}+\frac{t^{2}}{2 n} \varepsilon_{1}\left(\frac{t}{\sqrt{n}}\right),
$$

где $\left|\varepsilon_{1}(t)\right| \leqslant \beta^{3}$. Аналогично можно показать, что вторая производная функции $f$ допускает разложение:

$$
f^{\prime \prime}\left(\frac{t}{\sqrt{n}}\right)=-1+\frac{t}{\sqrt{n}} \varepsilon_{2}\left(\frac{t}{\sqrt{n}}\right)
$$

где также $\left|\varepsilon_{2}(t)\right| \leqslant \beta^{3}$. Используя эти выражения, мы получаем

$$
\begin{aligned}
h^{\prime}(t)= & t+\sqrt{n} \frac{f^{\prime}(t / \sqrt{n})}{f(t / \sqrt{n})}=\frac{\sqrt{n}}{f(t / \sqrt{n})}\left[\frac{t}{\sqrt{n}}\left(f\left(\frac{t}{\sqrt{n}}\right)-1\right)+\frac{t^{2}}{n} \varepsilon_{1}\left(\frac{t}{\sqrt{n}}\right)\right], \\
h^{\prime \prime}(t)= & 1+\frac{f^{\prime \prime}(t / \sqrt{n})}{f(t / \sqrt{n})}-\left[\frac{f^{\prime}(t / \sqrt{n})}{f(t / \sqrt{n})}\right]^{2}=\left(f\left(\frac{t}{\sqrt{n}}\right)\right)^{-1}\left[f\left(\frac{t}{\sqrt{n}}\right)-1+\frac{t}{\sqrt{n}} \varepsilon_{2}\left(\frac{t}{\sqrt{n}}\right)\right] \\
& -\left(f\left(\frac{t}{\sqrt{n}}\right)\right)^{-2}\left[\frac{t^{2}}{n}+\frac{t^{4}}{4 n^{2}}\left(\varepsilon_{1}\left(\frac{t}{\sqrt{n}}\right)\right)^{2}-\frac{t^{3}}{n^{3 / 2}} \varepsilon_{2}\left(\frac{t}{\sqrt{n}}\right)\right]
\end{aligned}
$$




$$
\begin{aligned}
h^{\prime \prime \prime}(t)= & \frac{1}{\sqrt{n}}\left[\frac{f^{\prime \prime \prime}(t / \sqrt{n})}{f(t / \sqrt{n})}-3 \frac{f^{\prime}(t / \sqrt{n}) f^{\prime \prime}(t / \sqrt{n})}{(f(t / \sqrt{n}))^{2}}+2\left(\frac{f^{\prime}(t / \sqrt{n})}{f(t / \sqrt{n})}\right)^{3}\right] \\
= & \frac{f^{\prime \prime \prime}(t / \sqrt{n})}{\sqrt{n} f(t / \sqrt{n})}-\frac{3}{\sqrt{n}(f(t / \sqrt{n}))^{2}} \\
& \times\left[-\frac{t}{\sqrt{n}}-\frac{t^{2}}{2 n} \varepsilon_{1}\left(\frac{t}{\sqrt{n}}\right)+\frac{t^{2}}{n} \varepsilon_{2}\left(\frac{t}{\sqrt{n}}\right)+\frac{t^{3}}{2 n^{3 / 2}} \varepsilon_{1}\left(\frac{t}{\sqrt{n}}\right) \varepsilon_{2}\left(\frac{t}{\sqrt{n}}\right)\right] \\
& +\frac{2}{\sqrt{n}(f(t / \sqrt{n}))^{3}}\left[-\frac{t^{3}}{n^{3 / 2}}+3 \frac{t^{4}}{2 n^{2}} \varepsilon_{1}\left(\frac{t}{\sqrt{n}}\right)+3 \frac{t^{5}}{4 n^{5 / 2}}\left(\varepsilon_{1}\left(\frac{t}{\sqrt{n}}\right)\right)^{2}\right. \\
& \left.\quad+\frac{t^{6}}{8 n^{3}}\left(\varepsilon_{1}\left(\frac{t}{\sqrt{n}}\right)\right)^{3}\right] .
\end{aligned}
$$

Используя (8) и неравенство $\left|f^{(k)}(t)\right| \leqslant \mathbf{E}\left|X_{1}\right|^{k}$, справедливое для всех $t$, из представления (9) мы получаем

$$
\left|h^{\prime}(t)\right| \leqslant\left|f\left(\frac{t}{\sqrt{n}}\right)\right|^{-1}\left[|t|\left|f\left(\frac{t}{\sqrt{n}}\right)-1\right|+\frac{t^{2}}{\sqrt{n}}\left|\varepsilon_{1}\left(\frac{t}{\sqrt{n}}\right)\right|\right] \leqslant 1.143\left[\frac{t^{4}}{2 n}+\frac{t^{2}}{\sqrt{n}} \beta^{3}\right] .
$$

Поэтому, так как $\beta^{3} \geqslant 1$ и $|t| \leqslant n^{1 / 6} / \beta$, то

$$
\left|h^{\prime}(t)\right| \leqslant 1.143 \frac{\beta^{3}}{\sqrt{n}} t^{2}+0.5715 \frac{\beta^{3}}{n^{5 / 6}} t^{2} .
$$

Аналогичным способом из (10)-(11) мы получаем

$$
\begin{aligned}
\left|h^{\prime \prime}(t)\right| & \leqslant 1.143 \frac{\beta^{3}}{\sqrt{n}}|t|+2.8579 \frac{\beta^{3}}{n^{5 / 6}}|t|, \\
\left|h^{\prime \prime \prime}(t)\right| & \leqslant 1.143 \frac{\beta^{3}}{\sqrt{n}}+21.8387 \frac{\beta^{3}}{n^{5 / 6}} .
\end{aligned}
$$

Далее, используя те же методы, из (12) и (13) получим оценки для произведений производных функции $h(t)$. Находим

$$
\begin{aligned}
\left|h^{\prime}(t)\right|^{2} & \leqslant 1.3065 \frac{\beta^{3}}{\sqrt{n}}|t|+1.6332 \frac{\beta^{3}}{n^{5 / 6}}|t|, \\
\left|h^{\prime}(t)\right|^{3} & \leqslant 1.4934 \frac{\beta^{3}}{\sqrt{n}}+3.5469 \frac{\beta^{3}}{n^{5 / 6}}, \\
\left|h^{\prime}(t) h^{\prime \prime}(t)\right| & \leqslant 1.3065 \frac{\beta^{3}}{\sqrt{n}}+5.5532 \frac{\beta^{3}}{n^{5 / 6}} .
\end{aligned}
$$

Из теоремы 8.9 в книге [5] вытекает, что

$$
|\exp \{h(t)\}|=e^{t^{2} / 2}|f(t / \sqrt{n})|^{n} \leqslant e^{t^{2} / 2} e^{-t^{2} / 3}=e^{t^{2} / 6} .
$$

Из теоремы 8.4 в книге [5] вытекает, что

$$
|\exp \{h(t)\}-1|=e^{t^{2} / 2}\left|f_{n}(t)-e^{-t^{2} / 2}\right| \leqslant 0.36 e^{t^{2} / 2} \frac{\beta^{3}}{\sqrt{n}}|t|^{3} \exp \left\{-0.3779 t^{2}\right\} .
$$

Таким образом, собирая оценки (12)-(19), мы получаем

$$
\begin{aligned}
& \left|f_{n}^{\prime \prime \prime}(t)-\left(3 t-t^{3}\right) e^{-t^{2} / 2}\right|=\left|\left(f_{n}(t)-e^{-t^{2} / 2}\right)^{\prime \prime \prime}\right| \\
& \leqslant \frac{\beta^{3}}{\sqrt{n}}\left[0.36\left(t^{6}+3 t^{4}\right) \exp \left\{-0.3779 t^{2}\right\}+\left(3.429 t^{4}+10.7775 t^{2}+6.5559\right) e^{-t^{2} / 3}\right] \\
& \quad+\frac{\beta^{3}}{n^{5 / 6}}\left(1.7145 t^{4}+15.1566 t^{2}+41.9552\right) e^{-t^{2} / 3} .
\end{aligned}
$$

Лемма доказана. 

$f_{n}(t)$ :

Доказательство те оремы 1 . Продифференпируем трижды х.ф.

$$
\begin{aligned}
f_{n}^{\prime \prime \prime}(t)= & \frac{(n-1)(n-2)}{\sqrt{n}} f^{n-3}\left(\frac{t}{\sqrt{n}}\right)\left(f^{\prime}\left(\frac{t}{\sqrt{n}}\right)\right)^{3}+ \\
& +3 \frac{(n-1)}{\sqrt{n}} f^{n-2}\left(\frac{t}{\sqrt{n}}\right) f^{\prime}\left(\frac{t}{\sqrt{n}}\right) f^{\prime \prime}\left(\frac{t}{\sqrt{n}}\right) \\
& +\frac{1}{\sqrt{n}} f^{n-1}\left(\frac{t}{\sqrt{n}}\right) f^{\prime \prime \prime}\left(\frac{t}{\sqrt{n}}\right) .
\end{aligned}
$$

Воспользовавшись оценкой $\left|f^{(k)}(t)\right| \leqslant \mathbf{E}\left|X_{1}\right|^{k}$, которая верна для любого $t$, легко получить неравенство $\left|f_{n}^{\prime \prime \prime}(t)\right| \leqslant\left(n^{2} \beta^{3} / \sqrt{n}\right)\left|f^{n-3}(t / \sqrt{n})\right|$. Поэтому при любом $n \geqslant 5$ по формуле Планшереля мы имеем

$$
\int_{-\infty}^{\infty}\left|f_{n}^{\prime \prime \prime}(t)\right| d t \leqslant\left(n^{2} \beta^{3}\right) \int_{-\infty}^{\infty}|f(t)|^{2} d t=2 \pi n^{2} \beta^{3} \int_{-\infty}^{\infty} p^{2}(x) d x \leqslant 2 \pi n^{2} \beta^{3} A<\infty .
$$

Следовательно, при указанных $n$ существует конечный интеграл от функции $f_{n}^{\prime \prime \prime}(t)$, и по формуле обращения преобразования Фурье мы имеем

$$
\begin{aligned}
2 \pi(i x)^{3} p_{n}(x) & =\int_{-\infty}^{\infty} e^{-i t x} f_{n}^{\prime \prime \prime}(t) d t \\
2 \pi(i x)^{3} \varphi(x) & =\int_{-\infty}^{\infty}\left(3 t-t^{3}\right) \exp \left\{-i t x-\frac{t^{2}}{2}\right\} d t
\end{aligned}
$$

Таким образом, при $n \geqslant 8 \beta^{6}$ с учетом неравенства $n^{1 / 6} / \beta \leqslant \sqrt{n} / 2 \beta^{3}$ мы получаем

$$
\begin{aligned}
& 2 \pi|x|^{3}\left|p_{n}(x)-\varphi(x)\right| \leqslant \int_{-\infty}^{\infty}\left|f_{n}^{\prime \prime \prime}(t)-\left(3 t-t^{3}\right) e^{-t^{2} / 2}\right| d t \\
& \leqslant \int_{|t| \leqslant n^{1 / 6} / \beta}\left|f_{n}^{\prime \prime \prime}(t)-\left(3 t-t^{3}\right) e^{-t^{2} / 2}\right| d t \\
& \quad+\int_{n^{1 / 6} / \beta<|t| \leqslant \sqrt{n} /\left(2 \beta^{3}\right)}\left|f_{n}^{\prime \prime \prime}(t)\right| d t+\int_{|t|>\sqrt{n} /\left(2 \beta^{3}\right)}\left|f_{n}^{\prime \prime \prime}(t)\right| d t \\
& \quad+\int_{|t|>n^{1 / 6} / \beta}\left(|t|^{3}+3|t|\right) e^{-t^{2} / 2} d t \equiv I_{1}+I_{2}+I_{3}+I_{4} .
\end{aligned}
$$

С помощью леммы 1 мы легко получаем следующую оценку для интеграла $I_{1}$ :

$$
\begin{aligned}
I_{1} \leqslant & 0.36 \frac{\beta^{3}}{\sqrt{n}} \int_{-\infty}^{\infty}\left(t^{6}+3 t^{4}\right) \exp \left\{-0.3779 t^{2}\right\} d t \\
& +\frac{\beta^{3}}{\sqrt{n}} \int_{-\infty}^{\infty}\left(3.429 t^{4}+10.7775 t^{2}+6.5559\right) e^{-t^{2} / 3} d t \\
& +\frac{\beta^{3}}{n^{5 / 6}} \int_{-\infty}^{\infty}\left(1.7145 t^{4}+15.1566 t^{2}+41.9552\right) e^{-t^{2} / 3} d t \\
\leqslant & 193.2302 \frac{\beta^{3}}{\sqrt{n}}+234.1259 \frac{\beta^{3}}{n^{5 / 6}} .
\end{aligned}
$$

Рассмотрим $I_{2}$. С помошью (20) имеем

$$
\left|f_{n}^{\prime \prime \prime}(t)\right| \leqslant \frac{\left|f_{n}(t)\right|}{\sqrt{n}}\left(\frac{(n-1)(n-2)}{|f(t / \sqrt{n})|^{3}}+\frac{3(n-1)}{|f(t / \sqrt{n})|^{2}}+\frac{\beta^{3}}{|f(t / \sqrt{n})|}\right) .
$$

Так как $|t| \leqslant \sqrt{n} /\left(2 \beta^{3}\right)$, а следовательно, и $|t| \leqslant \sqrt{n} / 2$, мы получаем

$$
\left|f\left(\frac{t}{\sqrt{n}}\right)-1\right| \leqslant \frac{t^{2}}{2 n} \leqslant \frac{1}{8}, \quad\left|f\left(\frac{t}{\sqrt{n}}\right)\right| \geqslant \frac{7}{8} .
$$


Из теоремы 8.9 в книге [5] следует, для любого $|t| \leqslant \sqrt{n} / 2 \beta^{3}$ имеет место оценка $\left|f_{n}(t)\right| \leqslant e^{-t^{2} / 3}$. Поэтому, применяя неравенства (22) и (23), для интеграла $I_{2}$ мы получаем оценку

$$
\begin{aligned}
I_{2} & \leqslant\left[\frac{512}{343} \frac{(n-1)(n-2)}{\sqrt{n}}+\frac{192}{49} \frac{(n-1)}{\sqrt{n}}+\frac{8}{7} \frac{\beta^{3}}{\sqrt{n}}\right] \int_{n^{1 / 6} / \beta<|t| \leqslant \sqrt{n} /\left(2 \beta^{3}\right)} e^{-t^{2} / 3} d t \\
& \leqslant 3 \sqrt{2 \pi} \frac{\beta}{n^{1 / 6}} \varphi\left(\sqrt{\frac{2}{3}} \frac{n^{1 / 6}}{\beta}\right)\left(1.4928 n^{3 / 2}+3.9184 n^{1 / 2}+1.1429 \frac{\beta^{3}}{\sqrt{n}}\right) \\
& \leqslant \beta \varphi\left(\sqrt{\frac{2}{3}} \frac{n^{1 / 6}}{\beta}\right)\left(11.2257 n^{4 / 3}+29.466 n^{1 / 3}+8.5945 \beta^{3} n^{-2 / 3}\right) .
\end{aligned}
$$

В работах Н.Г. Ушакова [6], [8] приведена теорема, из которой следует, что для х.ф. $f(t)$ при условиях существования третъего момента и ограниченной плотности для любого $\delta \in(0,1)$ справедливы неравенства

$$
\begin{array}{ll}
|f(t)| \leqslant 1-\frac{(1-\delta)^{3} t^{2}}{3 \pi^{2} A^{2}}, & |t| \leqslant \frac{\pi \delta^{1 / 3}}{2 \beta} ; \\
|f(t)| \leqslant 1-\frac{(1-\delta)^{3} \delta^{2 / 3}}{12 A^{2} \beta^{2}}, & |t|>\frac{\pi \delta^{1 / 3}}{2 \beta} .
\end{array}
$$

Заметим, что при любом $\delta \in\left(\pi^{-3}, 1\right)$, используя равенство (20), интеграл $I_{3}$ можно оценить следуюшим образом:

$$
\begin{aligned}
I_{3} \leqslant & \int_{1 /\left(2 \beta^{3}\right)<|t| \leqslant \pi \delta^{1 / 3} /(2 \beta)}\left[n^{2}|f(t)|^{n-3}+\beta^{3}|f(t)|^{n-1}\right] d t \\
& +\beta^{3} n^{2} \int_{|t|>\pi \delta^{1 / 3} /(2 \beta)}|f(t)|^{n-3} d t \equiv I_{31}+I_{32} .
\end{aligned}
$$

Воспользовавшись неравенством (25), получим

$$
\begin{aligned}
I_{31} \leqslant 2 \beta^{3} \int_{1 /\left(2 \beta^{3}\right)^{-1}<|t| \leqslant \pi \delta^{1 / 3} /(2 \beta)}|t| \\
\quad \times\left[n^{2}\left(1-\frac{(1-\delta)^{3} t^{2}}{3 \pi^{2} A^{2}}\right)^{n-3}+\beta^{3}\left(1-\frac{(1-\delta)^{3} t^{2}}{3 \pi^{2} A^{2}}\right)^{n-1}\right] d t \\
\quad \leqslant 2 \beta^{3} \int_{1 /\left(4 \beta^{6}\right)^{-1}}^{\pi^{2} \delta^{2 / 3} /\left(4 \beta^{2}\right)}\left[n^{2}\left(1-\frac{(1-\delta)^{3} u}{3 \pi^{2} A^{2}}\right)^{n-3}+\beta^{3}\left(1-\frac{(1-\delta)^{3} u}{3 \pi^{2} A^{2}}\right)^{n-1}\right] d u \\
=\frac{6 A^{2} \pi^{2} \beta^{3}}{(1-\delta)^{3}} \int_{0}^{1-(1-\delta)^{3} /\left(12 A^{2} \pi^{2} \beta^{6}\right)}\left[n^{2} z^{n-3}+\beta^{3} z^{n-1}\right] d z \\
\leqslant \frac{6 A^{2} \pi^{2} \beta^{3}}{(1-\delta)^{3}}\left[1.34 n q_{1}^{n-2}+\frac{\beta^{3}}{n} q_{1}^{n}\right]
\end{aligned}
$$

где $q_{1}=1-(1-\delta)^{3} /\left(12 \pi^{2} A^{2} \beta^{6}\right)$. Для любого $n$ при произвольном $q \in(0,1)$ число

$$
M=\sup _{x \geqslant 0} x^{\alpha} q^{x(1-\gamma)} \geqslant \sup _{n \geqslant 1} n^{\alpha} q^{n(1-\gamma)}
$$

гарантирует выполнение соотношения $n^{\alpha} q^{n} \leqslant M q^{\gamma n}$ для любых $\alpha>0$ и $\gamma \in(0,1)$. Элементарными вычислениями находим $M=[\alpha /(e(1-\gamma)|\ln q|)]^{\alpha} \leqslant[\alpha /(e(1-\gamma) \times$ $(1-q))]^{\alpha}$. Далее, несложно видеть, что $q_{1}^{-1} \leqslant 1.1128$. Следовательно, полагая $\alpha=1$ и $q=q_{1}$, из (27) мы получаем неравенство

$$
\begin{aligned}
I_{31} & \leqslant \frac{6 A^{2} \pi^{2} \beta^{3}}{(1-\delta)^{3}}\left[\frac{7.3254 \pi^{2} A^{2} \beta^{6}}{(1-\gamma)(1-\delta)^{3}} q_{1}^{\gamma n}+\frac{1.22 \pi^{2} A^{2} \beta^{6}}{(1-\gamma)(1-\delta)^{3}} q_{1}^{\gamma n}\right] \\
& \leqslant 4995 \frac{A^{4} \beta^{9}}{(1-\gamma)(1-\delta)^{6}} q_{1}^{\gamma n} .
\end{aligned}
$$


В то же время, положив $q_{2}=1-(1-\delta)^{3} \delta^{2 / 3} /\left(12 A^{2} \beta^{2}\right)$ и используя неравенство (26) и формулу Планшереля, мы будем иметь

$$
\begin{aligned}
I_{32} & =\beta^{3} n^{2} \int_{|t|>\pi \delta^{1 / 3} /(2 \beta)}|f(t)|^{2}|f(t)|^{n-5} d t \\
& \leqslant 2 \pi \beta^{3} n^{2} q_{2}^{n-5} \int_{-\infty}^{\infty}|p(x)|^{2} d x \leqslant 2 \pi A \beta^{3} n^{2} q_{2}^{n-5} .
\end{aligned}
$$

Можно показать, что $q_{2}^{-1} \leqslant 1.22$. Используя определение чпсла $M$ с $\alpha=2$, получаем

$$
\begin{aligned}
I_{32} & \leqslant 2(1.22)^{5} \pi A \beta^{3}\left(\frac{24 A^{2} \beta^{2}}{e(1-\gamma)(1-\delta)^{3} \delta^{2 / 3}}\right)^{2} q_{2}^{\gamma n} \\
& \leqslant 1324 \frac{A^{5} \beta^{7}}{(1-\gamma)^{2}(1-\delta)^{6} \delta^{4 / 3}} q_{2}^{\gamma n} .
\end{aligned}
$$

Заметим, что в наших условиях $\delta \in\left(\pi^{-3}, 1\right)$, а в данном интервале верно неравенство $q_{2} \leqslant q_{1}$. С учетом данного замечания, объединив оценки (28) и (29), получим

$$
I_{3} \leqslant \frac{A^{4} \beta^{9}}{(1-\gamma)(1-\delta)^{6}}\left[4995+1324 \frac{A}{(1-\gamma) \delta^{4 / 3}}\right]\left(1-\frac{(1-\delta)^{3}}{12 \pi^{2} A^{2} \beta^{6}}\right)^{\gamma n} .
$$

Рассмотрим $I_{4}$. Имеем

$$
I_{4} \leqslant \int_{|t|>n^{1 / 6} / \beta} t^{3} e^{-t^{2} / 2} d t+3 \int_{|t|>n^{1 / 6} / \beta} t e^{-t^{2} / 2} d t \equiv I_{41}+I_{42} .
$$

При этом $I_{41}=10.0266 \varphi\left(n^{1 / 6} / \beta\right)\left(n^{1 / 3} / 2+1\right), I_{42} \leqslant 15.0399 \varphi\left(n^{1 / 6} / \beta\right)$. Таким образом, получаем

$$
\begin{aligned}
I_{4} & \leqslant \varphi\left(\frac{n^{1 / 6}}{\beta}\right)\left(5.0133 n^{1 / 3}+25.0665\right) \\
& \leqslant \beta \varphi\left(\sqrt{\frac{2}{3}} \frac{n^{1 / 6}}{\beta}\right)\left(5.0133 n^{1 / 3}+25.0665\right) .
\end{aligned}
$$

Суммируя оценки (21), (24), (30) и (31), получаем требуемое утверждение.

Из теоремы 1 вытекает следующая асимптотическая оценка абсолютной константы в неравномерной оценке скорости сходимости в локальной предельной теореме для плотностей.

Следствие 1. В условиях теоремы 1 при всех $x \in \mathbf{R}$

$$
\limsup _{n \rightarrow \infty} \frac{\sqrt{n}}{\beta^{3}}|x|^{3} \Delta(x) \leqslant 30.7536 .
$$

В работе [9] была получена аналогичная оценка абсолютной константы в равномерной оценке скорости сходимости. В сделанных нами предположениях верно неравенство

$$
\lim _{n \rightarrow \infty} \frac{\sqrt{n}}{\beta^{3}} \sup _{x}\left|p_{n}(x)-\varphi(x)\right| \leqslant 0.4013 .
$$

Заметим, что абсолютные константы в оценках (32) и (33) и аналогичные величины в интегральной центральной предельной теореме довольно мало отличаются друг от друга.

Далее, с помощью рассуждений, аналогичных тем, что использовались при доказательстве теоремы 1 , мы можем получить неравномерную оценку порядка $|x|$, по структуре аналогичную неравенству (5), но с менъшей константой, что позволяет надеяться на улучшение оценки при умеренных значениях $|x|$ за счет меньшей константы.

Теорема 2. В условиях теоремы 1 имеет место неравенство

$$
|x| \Delta(x) \leqslant 1.7054 \frac{\beta^{3}}{\sqrt{n}}+\Delta_{n}^{(7)}(\beta)+\Delta_{n}^{(8)}(A, \beta),
$$


¿de

$$
\begin{aligned}
& \Delta_{n}^{(7)}(\beta)=0.4189 \frac{\beta^{3}}{n^{5 / 6}}+\beta \varphi\left(\sqrt{\frac{2}{3}} \frac{n^{1 / 6}}{\beta}\right)\left(1.3679 n^{1 / 3}+0.7979\right),
\end{aligned}
$$

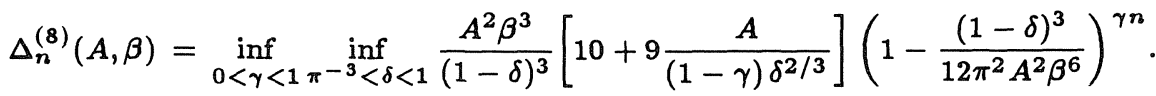

С помошью леммы 1 можно показать, что оценка для величины $x^{2} \Delta(x)$, полученная в работе [9] для симметричных слагаемых, остается верной и для общего случая. Поэтому, используя эту оценку, а также неравенства (2) и (5), мы можем уточнить оценку для $\left\|p_{n}(x)-\varphi(x)\right\|_{p}$, полученную в [9].

Теорема 3. В условиях теоремы 1 при $p \geqslant 1$ справедлива оченка

$$
\begin{aligned}
\left\|p_{n}(x)-\varphi(x)\right\|_{p} \leqslant & C_{1}(p) \frac{\beta^{3}}{\sqrt{n}}+C_{2}(p)\left[\Delta_{n}^{(1)}(\beta)+\Delta_{n}^{(2)}(A, \beta)\right] \\
& +C_{3}(p)\left[\Delta_{n}^{(3)}(\beta)+\Delta_{n}^{(4)}(A, \beta)\right]+C_{4}(p)\left[\Delta_{n}^{(5)}(\beta)+\Delta_{n}^{(6)}(A, \beta)\right],
\end{aligned}
$$

2de

$$
\begin{aligned}
\Delta_{n}^{(3)}(\beta)= & 3.7817 \frac{\beta^{3}}{n^{5 / 6}}+\beta \varphi\left(\sqrt{\frac{2}{3}} \frac{n^{1 / 6}}{\beta}\right)\left(1.5633 n^{5 / 6}+0.7979 n^{1 / 6}+3.7617 n^{-1 / 6}\right) \\
\Delta_{n}^{(4)}(A, \beta)= & \inf _{0<\gamma<1} \inf _{\pi^{-3}<\delta<1} \frac{A^{3} \beta^{3}}{\sqrt{1-\gamma}(1-\delta)^{9 / 2}}\left[79 \beta^{3}+38 \frac{A}{\delta(1-\gamma)}\right] \\
& \times\left(1-\frac{(1-\delta)^{3}}{12 \pi^{2} A^{2} \beta^{6}}\right)^{\gamma n}, \\
C_{1}(p)= & \left(0.4013^{p} \frac{16.6686 p}{2 p-1}-2 \cdot 0.2265^{3 p-1} \frac{p}{6 p^{2}-5 p+1}\right)^{1 / p} \\
C_{2}(p)= & 8.3343^{1 / p}, \quad C_{3}(p)=\left(\frac{0.0576^{p-1 / 2}}{2 p-1}-\frac{0.2265^{2 p-1}}{2 p-1}\right)^{1 / p} \\
C_{4}(p)= & \left(\frac{0.2265^{3 p-1}}{3 p-1}\right)^{\frac{1}{p}} .
\end{aligned}
$$

Наконец, из теоремы 3 мы получаем следующую асимптотическую оценку константы в оценке скорости сходимости в локальной центральной предельной теореме для плотностей в терминах метрики $L_{p}$.

Следствие 2. В условиях теоремы 3 при всех $x \in \mathbf{R}$

$$
\limsup _{n \rightarrow \infty} \frac{\sqrt{n}}{\beta^{3}}\left\|p_{n}(x)-\varphi(x)\right\|_{p} \leqslant C_{1}(p),
$$

где $C_{1}(p)$ определено в (35).

В частности,

$$
C_{1}(1) \leqslant 6.6378, \quad C_{1}(2) \leqslant 1.3377, \quad C_{1}(3) \leqslant 0.8647 .
$$

В заключение автор выражает признательность Ю.В. Прохорову, внимательно прочитавшему первоначальный вариант рукописи и указавшему на некоторые неточности, а также В.Ю. Королеву за постоянное внимание к работе.

\section{СПИСОК ЛИТЕРАТУРЫ}

1. Сираждинов C. X., Шахайдарова $H$. О равномерной локальной теореме для плотностей. - Изв. АН У

2. Шахайдарова $H$. Равномерные локальные и глобальные теоремы для плотностей. - Изв. АН УзССР, сер. физ.-матем., 1966, № 5, с. 90-91.

3. Петров В.В. Суммы независимых случайных величин. М.: Наука, 1972, 416 с. 
4. Прохоров ЮО.В. Об одной лохальной теореме. - В сб.: Предельные теоремы теории вероятностей. Ташкент: Изд-во АН УзССР, 1963, с. 75-80.

5. Бхаттачария $P$. H., Ранга $Р$ ао $P$. Аппроксимация нормальным распределением и асимптотические разложения. М.: Наука, 1982, 286 с.

6. Ушаков Н.Г. Неравенства для характеристических функпий. - В сб.: Методы решения задач математической физики и их программное обеспечение. М.: Изд-во Моск. ун-та, 1984, с. 50-53.

7. Феллер В. Введение в теорию вероятностей и ее приложения. Т. 2. М.: Мир, 1984, $752 \mathrm{c}$.

8. Ushakov N. G. Lower and upper bounds for characteristic functions. - J. Math. Sci., 1997 , v. 84, № 3, p. 1179-1189.

9. Korolev V. Yu., Zhukov Yu. V. On the rate of convergence in the local limit theorem for densities. - J. Math. Sci., 1998, v. 91, № 3, p. 2931-2941.

Поступила в редакцию 19.I.1998

(C) $1999 \mathrm{r}$.

ЗУБКОВ А. M.*

\title{
ИЗОМОРФИЗМЫ МЕЖДУ МНОЖЕСТВАМИ ВЕРОЯТНОСТНЫХ РАСПРЕДЕЛЕНИЙ ${ }^{1)}$
}

\begin{abstract}
Указаны способы построения изоморфизмов между полугруппами вероятностных распределений (со сверткой как полугрупповой операцией), в частности, между множеством всех вероятностных распределений на неотрицательной полуоси и некоторой совокупностью распределений на множестве неотрицательных целых чисел. Аналогичные изоморфизмы указаны для векторного случая и для множеств распределений, соответствуюших неубывающим процессам с независимыми приращениями.
\end{abstract}

Ключевые слова и фразы: полугруппы вероятностных распределений, неотрицательные случайные величины, изоморфизмы между множествами распределений.

В настоящей работе используется следуюшее соображение. Пусть $P$ - семейство всех вероятностных распределений на $\mathbf{R}_{+}=[0, \infty)$. Обозначим через $\delta_{x}$ распределение, сосредоточенное в точке $x \in \mathbf{R}_{+}$, и через $D=\left\{\delta_{x}, x \in \mathbf{R}_{+}\right\}$семейство вырожденных распределений на $\mathbf{R}_{+}$. Относительно операции свертки семейства $P$ и $D$ являются коммутативными полугруппами; более того, для однопараметрического семейства $D$ полугрупповая структура, порожденная сверткой, согласована со структурой аддитивной полугруппы $\mathbf{R}_{+}: \delta_{x} * \delta_{y}=\delta_{x+y}$ при любых $x, y \in \mathbf{R}_{+}$.

Любое вероятностное распределение $F \in P$ можно представить в виде смеси распределений из семейства $D$ :

$$
F=\int_{0}^{\infty} \delta_{x} F(d x)
$$

Пусть, далее, $B=\left\{B_{x}, x \in \mathbf{R}_{+}\right\}-$любое семейство распределений (например, на $\mathbf{R}^{d}$ ), образующее полугруппу относительно операции свертки, причем

* Математический институт им. В. А. Стеклова РАН, ул. Губкина, 8, 117966 Москва, ГСП-1, Россия; e-mail: zubkov@genesis.mi.ras.ru

1) Выполнение работы частично финансировалось Российским фондом фундаментальных исследований (проект 96-01-00531) и Советом по грантам Президента РФ и государственной поддержке ведуших научных школ (проект 96-15-96092). 